\title{
Tumor neuroectodérmico primitivo periférico originado em ramo do nervo ciático
}

\author{
Roberto S. Martins*, Mario G. Siqueira*, Osmar J. S. Moraes*, \\ João N. Stavale**, Nise Yamaguchi*** \\ Centro Integrado de Neurocirurgia. Hospital Beneficência Portuguesa. São Paulo, SP
}

\section{RESUMO}

Os autores relatam caso de tumor neuroectodérmico primitivo periférico originado de ramo do nervo ciático, em paciente de 17 anos, do sexo feminino. O tumor foi extirpado cirurgicamente e o tratamento foi complementado com rádio e quimioterapia. A lesão apresentava tanto um aspecto histológico característico, com células pequenas e arredondadas formando pseudo-rosetas, como um perfil imunoistoquímico compatível.

Os tumores neuroectodérmicos primitivos de nervos periféricos são lesões raras, descritas em 36 casos da literatura. Neste relato são discutidas as principais características dessa lesão e enfatizada a importância de um tratamento cirúrgico agressivo associado à terapêutica adjuvante apropriada.

\section{PALAVRAS-CHAVE}

Nervo periférico. Tumor neuroectodérmico primitivo.

\section{ABSTRACT well as a compatible immunohistochemical profile. \\ KEYWORDS \\ Peripheral nerve. Primitive neuroectodermal tumor.}

Peripheral primitive neuroectodermal tumor arising in a branch of the sciatic nerve Primitive neuroectodermal tumors of peripheral nerves are rare lesions, existing 36 cases reported in the literature. The authors report a case of peripheral primitive neuroectodermal tumor arising in a branch of the sciatic nerve, in a 17 year-old female patient. The tumor was surgically extirpated and the treatment was complemented by radio and chemotherapy. The lesion presented a characteristic histologycal appearance, with small and rounded cells forming pseudorosets, as

In this report the main characteristics of these lesions are discussed and the importance of an aggressive surgical treatment, associated with an appropriated adjuvant therapy is emphasized.

\section{Introdução}

Os tumores neuroectodérmicos primitivos são neoplasias malignas cuja localização em nervos periféricos é rara, existindo somente 36 casos relatados na literatura. A análise imunoistoquímica em geral evidencia antígenos sugestivos de origem neural e foi descrita em oito casos.

Neste relato, é apresentado o caso de uma paciente de 17 anos com diagnóstico de tumor neuroectodérmico primitivo de nervo periférico do membro inferior submetido a tratamento cirúrgico, complementado por rádio e quimioterapia.

\section{Relato do caso}

A paciente Y. J. P., de 17 anos de idade, do sexo feminino, apresentou-se em março de 1999 com queixa, de um mês de duração, de dor na região posterior da coxa direita, com eventuais parestesias em formigamento que se estendiam ao quarto e quinto pododáctilos, associada a um abaulamento no local. O exame físico demonstrava um abaulamento na região posterior do terço médio da coxa direita, além de dor à palpação profunda local provocada por uma massa de contornos mal definidos. O exame neurológico fora normal.

\footnotetext{
*Neurocirurgiões do Centro Integrado de Neurocirurgia, Hospital Beneficência Portuguesa. São Paulo, SP. **Patologista do Departamento de Patologia da Universidade Federal de São Paulo.

*** Oncologista do Instituto Avanços em Medicina.
} 
A investigação pela ressonância magnética demonstrou uma formação expansiva sólida, com diâmetros aproximados de 7,0 x 5,5 centímetros, no terço médio da coxa, situada profundamente à massa muscular posterior da região, entre os músculos adutor magno e bíceps femoral. Bem delimitada e com hipersinal em todas as seqüências estudadas, a lesão apresentava realce intenso e homogêneo após a administração endovenosa de contraste paramagnético (Figura 1).

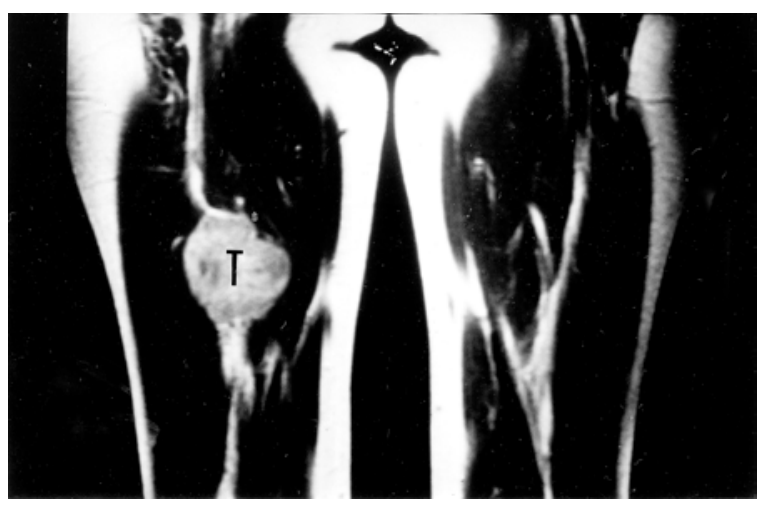

Figura 1 - Ressonância magnética dos membros inferiores, corte coronal, aquisição T1, evidenciando a lesão expansiva bem delimitada (T) e com realce intenso e homogêneo após a administração endovenosa de contraste paramagnético.

Com o diagnóstico provável de uma lesão expansiva de nervo periférico, a paciente foi submetida a tratamento cirúrgico através de uma incisão longitudinal mediana na face posterior da coxa. Profundamente ao plano muscular, identificou-se o nervo ciático deslocado posteriormente, sobre uma lesão de contornos irregulares, com cerca de 8 centímetros de diâmetro no seu maior eixo (Figura 2). Havia um plano de clivagem bem definido entre a lesão, o nervo e tecidos adjacentes, à exceção da região mais anterior, onde o tumor infiltrava a musculatura adjacente. Na porção mais proximal do campo operatório, foi isolado um delgado ramo profundo do nervo ciático, que dava origem à lesão expansiva. Após a exérese macroscópica completa da lesão, que incluiu ressecção da musculatura infiltrada, a confirmação de ausência de tumor foi determinada através de múltiplas biópsias por congelação da musculatura nos limites da ressecção. A análise microscópica da lesão demonstrou a presença de células pequenas com núcleos hipercromáticos e figuras de mitose, além de pseudo-rosetas (Figura 3), quadro histológico compatível com tumor neuroectodérmico primitivo. No exame imunoistoquímico, foram pesquisados os antígenos proteína ácida gliofibrilar, actina músculo-específica $(\mathrm{HHF} / 35)$, citoqueratinas (AE1/AE3), antimúsculo liso específico, cromogramina e MIC-2, pela técnica da strepta-avidina-biotinaperoxidase. Houve imunoexpressão discreta para cromogramina e forte para MIC-2 nas células neoplásicas, configurando um padrão imunofenotípico de neoplasia de células neurais primitivas.

A

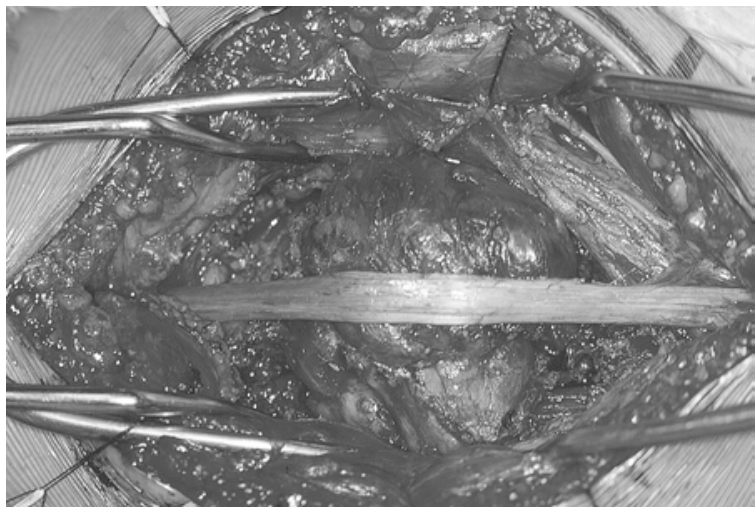

B

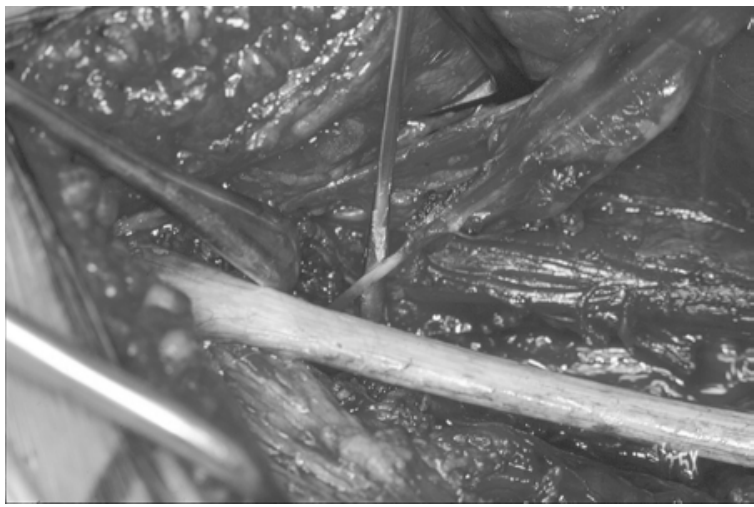

Figura 2 - A) Fotografia cirúrgica da lesão expansiva deslocando posteriormente o nervo ciático.

B) Fotografia cirúrgica demonstrando delgado ramo profundo do nervo ciático, erguido por dissector, que dava origem à lesão expansiva.

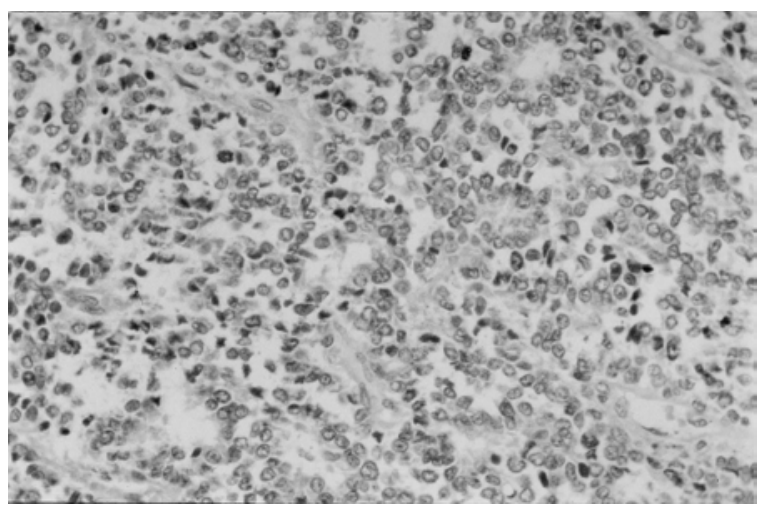

Figura 3 - Fotomicrografia da lesão expansiva: neoplasia de pequenas células com núcleos hipercromáticos e figuras de mitoses. Presença de pseudo-rosetas (H\&E, $400 \mathrm{X})$ 
O pós-operatório decorreu sem intercorrências, persistindo a normalidade do exame neurológico. A possível ocorrência de lesões múltiplas foi descartada através da realização de tomografia computadorizada de tórax e ressonância magnética de abdome, crânio e coluna vertebral. A paciente recebeu tratamento adjuvante sob a forma de quimioterapia (ifosfamida e paraplatim) e radioterapia local com dose total de $5.400 \mathrm{cGy}$. Com 18 meses de seguimento pós-operatório, a paciente não apresenta quaisquer queixas e não existem evidências clínicas ou por imagem de recorrência da doença.

\section{Discussão}

Os tumores neuroectodérmicos primitivos são neoplasias malignas que ocorrem com maior freqüência em crianças e que apresentam localização preferencialmente intracraniana. No cerebelo, onde incidem com maior freqüência, recebem a denominação de meduloblastomas. Podem ocorrer também na região da glândula pineal, no parênquima cerebral, no tronco cerebral, na medula espinhal, na glândula supra-renal e em nervos periféricos ${ }^{2,17}$. Os tumores neuroectodérmicos primitivos de nervos periféricos já receberam várias denominações, tais como neuroepitelioma periférico, meduloepitelioma e neuroblastoma periférico e, para complicar ainda mais o diagnóstico, podem apresentar grande semelhança histológica com sarcoma de Ewing, com neuroblastomas, rabdomiossarcomas e linfomas.

$\mathrm{O}$ primeiro autor a descrever um caso bem documentado de tumor neuroectodérmico primitivo em nervo periférico foi Stout, em 1918, existindo, atualmente, 36 casos relatados na literatura ${ }^{1,3-16,18-22}$. Histologicamente, esses tumores são caracterizados pela presença de células pequenas, esféricas ou ovais, homogêneas e de citoplasma basofílico. A atividade mitótica é intensa e células binucleadas podem estar presentes. A ocorrência de pseudo-rosetas (rosetas de Homer-Wright) é obrigatória para o diagnóstico histológico ${ }^{12}$. Outra apresentação característica é a presença de um padrão de fibras reticulares preenchendo o interstício, circundando e envolvendo as células neoplásicas. $\mathrm{Na}$ análise por microscopia eletrônica, as células tumorais apresentam expansões citoplasmáticas que se interdigitam e contêm grânulos neurosecretores ${ }^{3,9,10,12,13}$. Segundo Jürgens ${ }^{10}$, esses grânulos constituem um fator de diferenciação entre o sarcoma de Ewing e o tumor neuroectodérmico primitivo de nervos periféricos. $\mathrm{Na}$ análise imunoistoquímica, é demonstrada a presença de antígenos sugestivos da origem neuroectodérmica ${ }^{1,8,10,12,13}$. Nos nove casos da literatura em que esse estudo foi realizado ocorreu positividade para enolase neuronal específica em oito casos e ausência de positividade para sinaptosina em todos os casos, à exceção de um. A ausência de impregnação pela proteína fibrilar glial e a positividade para o marcador MIC2 também são características dessa patologia.

Clinicamente, este tipo de neoplasia pode ocorrer tanto em adultos como em crianças, sendo mais freqüente no primeiro grupo. Na literatura, a média de idade dos pacientes na época do diagnóstico é de 28,2 anos e, a média de sobrevida após o tratamento, de 11, 6 meses $^{1}$. É importante que, na investigação inicial, sejam descartadas as possibilidades de metástases pulmonares e hepáticas ${ }^{22}$.

Relatos recentes de pacientes portadores de tumor neuroectodérmico primitivo localizado fora do sistema nervoso central apresentam um prognóstico reservado e sugerem a necessidade de implantação de uma terapêutica agressiva no momento do diagnóstico ${ }^{8,10-13}$. A tendência desses tumores de recorrer localmente justifica uma cirurgia o mais radical possível numa primeira abordagem ${ }^{10}$. Devido ao reduzido número de casos publicados, não é possível determinar o tratamento ideal para os tumores neuroectodérmicos primitivos em nervos periféricos; no entanto, com base no tratamento desses tumores em outras localizações, é recomendável o emprego de uma conduta cirúrgica a mais radical possível, seguida de tratamento adjuvante sob a forma de rádio e quimioterapia. No caso relatado, a exérese cirúrgica radical foi confirmada através da ausência de lesão neoplásica nas biópsias por congelação de fragmentos de tecido da periferia do campo cirúrgico e nos estudos por imagem pós-operatórios. O tratamento cirúrgico, associado a rádio e quimioterapia, já propiciou à paciente uma sobrevida, livre de doença acima da média esperada, referida na literatura.

\section{Referências}

1. AKEYSON EW, MCCUTCHEON IE, PERSHOUSE MA, STECK PA, FULLER GN: Primitive neuroectodermal tumor of the median nerve. Case report with cytogenetic analysis. J Neurosurg 85:163-9, 1996.

2. BECKER LE, HINTON D: Primitive neuroectodermal tumors of the central nervous system. Hum Pathol 14:538-50, 1983

3. BOLEN JW, THORNING D: Peripheral neuroepithelioma: a light and electron microscopic study. Cancer 46:2456-62, 1980.

4. COHN I: Epithelial neoplasms of peripheral and cranial nerves. Report of three cases, review of the literature. Arch Surg 17:117-60, 1928.

5. HACKEL W: Zur frage über das medulloepitheliom. Beitr Pathol Anat Allg Pathol 92:510-7, 1934.

6. HARKIN JC: Differential diagnosis of peripheral nerve tumors. In Omer G, Spinner M (eds): Management of peripheral nerve lesions. Philadelphia, WB Saunders, 1980, pp 657-68. 
7. HARPER PG, PRINGLE J, SOUHAMI RL: Neuroepithelioma - a rare malignant peripheral nerve tumor of primitive origin: report of two new cases and a review of the literature. Cancer 48:2282-7, 1981.

8. HASHIMOTO H, KIRYU H, ENJOJI M: Malignant neuroepithelioma (peripheral neuroblastoma). A clinicopathologic study of 15 cases. Am J Surg Pathol 7:309-18, 1983

9. ISHIKAWA S, OSHIMA Y, SUZUKI T, OBOSHI S: Primitive neuroectodermal tumor (neuroepithelioma) of spinal nerve root - report of an adult case and stablishment of a cell line. Acta Pathol Jpn 29:289301, 1979

10. JÜRGENS H, BIER V, HARMS D, BECK J, BRANDEIS W, ETSPÜLER G, GARDNER H, SCHMIDT D, TREUNER J, WINKLER K, GÖBEL U: Malignant peripheral neuroectodermal tumors. A retrospective analysis of 42 patients. Cancer 61:349-57, 1988.

11. LAGERKVIST B, IVEMARK B, SYLVÉN B: Malignant neuroepithelioma in childhood. A report of three cases. Acta Chir Scand 135:641-5, 1969.

12. LLOMBART-BOSCH A, TERRIER-LACOMBE MJ, PEYDRO-OLAYA A, CONTESSO G: Peripheral neuroectodermal sarcoma of soft tissue (peripheral neuroepithelioma): a pathologic study of ten cases with differential diagnosis regarding other small, round-cell sarcomas. Hum Pathol 20:273-80, 1989.

13. MARINA NM, ETCUBANAS E, PARHAM DM: Peripheral primitive neuroectodermal tumor (peripheral neuroepithelioma) in children. A review of the St. Jude experience and controversies in diagnosis and management. Cancer 64:1952-60, 1989.

14. MENNEL HD, ZÜLCH KJ: Die morphologie maligner tumorens des peripheren nerven. Zentralbl Neurochir 32:11-24, 1971.
15. NAKAMURA Y, BECKER LE, MANCER K, GILLESPIE R: Peripheral medulloepithelioma. Acta Neuropathol (Berl) 57:137-42, 1982.

16. NESBITT KA, VIDONE RA: Primitive neuroectodermal tumor (neuroblastoma) arising in sciatic nerve of a child. Cancer 37:1562-70, 1976.

17. RORKE LB. The cerebellar medulloblastoma and its relationship to primitive neuroectodermal tumors. J Neuropath Exp Neurol 42:1-15, 1983.

18. SAMUEL AW: Primitive neuroectodermal tumor arising in the ulnar nerve. A case report. Clin Orthop Rel Res 167:236-8, 1982.

19. STOUT AP: A tumor of the ulnar nerve. Proc $N$ Y Pathol Soc 18:2-11, 1918.

20. STOUT AP, MURRAY MD: Neuroepithelioma of the radial nerve with a study of its behaviour in vitro. Rev Canad Biol 1:651-9, 1942.

21. STOUT AP: Tumors of the peripheral nervous system. J Missouri St Med Assoc 46: 255-9, 1949.

22. VOSS BL, PYSHER TJ, HUMPHREY GB: Peripheral neuroepithelioma in childhood. Cancer 54:3059-64, 1984.

Original recebido em janeiro de 2001

Aceito para publicação em setembro de 2001

\section{Endereço para correspondência:}

Roberto S. Martins

Centro Integrado de Neurocirurgia

Rua Maestro Cardim, 592 - cj. 1101

CEP 01323-001 - São Paulo, SP 